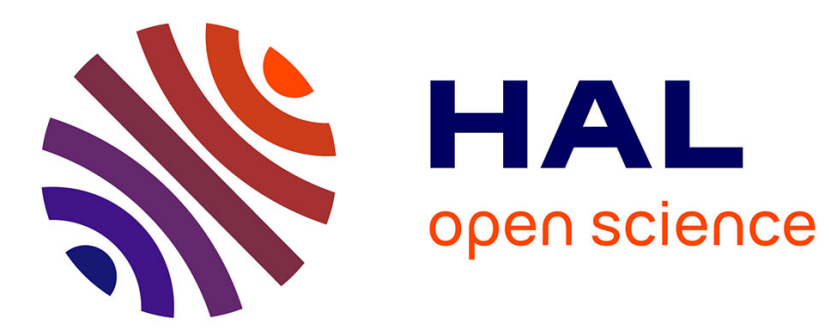

\title{
Désir et imitation
}

Sébastien Charbonnier

\section{To cite this version:}

Sébastien Charbonnier. Désir et imitation. Le Télémaque. Philosophie, Education, Société, 2012, 41

(1), 10.3917/tele.041.0009 . hal-01663460

\section{HAL Id: hal-01663460 \\ https://hal.science/hal-01663460}

Submitted on 13 Dec 2017

HAL is a multi-disciplinary open access archive for the deposit and dissemination of scientific research documents, whether they are published or not. The documents may come from teaching and research institutions in France or abroad, or from public or private research centers.
L'archive ouverte pluridisciplinaire HAL, est destinée au dépôt et à la diffusion de documents scientifiques de niveau recherche, publiés ou non, émanant des établissements d'enseignement et de recherche français ou étrangers, des laboratoires publics ou privés. 


\section{DÉSIR ET IMITATION}

Sébastien Charbonnier

Presses universitaires de Caen | «Le Télémaque »

2012/1 n 41 | pages 9 à 18

ISSN 1263-588X

ISBN 9782841334087

Article disponible en ligne à l'adresse :

https://www.cairn.info/revue-le-telemaque-2012-1-page-9.htm

\section{Pour citer cet article :}

Sébastien Charbonnier, «Désir et imitation 》, Le Télémaque 2012/1 (n41), p. 9-18. DOI 10.3917/tele.041.0009

Distribution électronique Cairn.info pour Presses universitaires de Caen.

(C) Presses universitaires de Caen. Tous droits réservés pour tous pays.

La reproduction ou représentation de cet article, notamment par photocopie, n'est autorisée que dans les limites des conditions générales d'utilisation du site ou, le cas échéant, des conditions générales de la licence souscrite par votre établissement. Toute autre reproduction ou représentation, en tout ou partie, sous quelque forme et de quelque manière que ce soit, est interdite sauf accord préalable et écrit de l'éditeur, en dehors des cas prévus par la législation en vigueur en France. Il est précisé que son stockage dans une base de données est également interdit. 


\section{CHRONIQUE MORALE}

\section{Désir et imitation}

Résumé: L'éducation met en relation les individus. Traditionnellement, on conçoit celle-là selon un schéma asymétrique: certains apprennent à d'autres, ces derniers apprennent des premiers. Mais si on se demande ce qui est premièrement en jeu dans l'éducation, c'est moins une problématique de transmission qu'une problématique formative: comment mettre en branle les énergies des individus pour qu'ils se forment - eux-mêmes et entre eux? Ce qui est au cour de la relation éducative, c'est d'abord le désir et ses conditions de circulation. Cette priorité ontologique n'a pas qu'une visée éthique: elle fonde pareillement les conditions épistémologiques de construction du savoir au sein de l'apprentissage. Le vrai problème de l'éducation est donc: comment partager avec l'autre mon désir de connaître pour que nous puissions apprendre des choses l'un de l'autre en participant à une communauté de recherche. Pour explorer ce problème, l'auteur s'appuie sur Spinoza, Gabriel Tarde et René Girard: trois grands penseurs du rapport entre désir et imitation. En essayant d'articuler leurs analyses, qui se complètent plus qu'elles ne se contredisent, il est possible de comprendre la dynamique du désir: son réquisit d'égalité épistémique; sa puissance transindividuelle de modifier, de part et d'autre, les acteurs du geste éducatif; l'horizon proprement politique de convenance qu'elle permet d'instituer entre les individus.

Le désir et l'imitation forment l'articulation même entre le collectif et les individus et nous placent ainsi au cœur des questions d'éducation. Comment partager un savoir, une valeur qu'on croit désirable et dont on voudrait qu'il ou elle soit pareillement désirable pour les autres individus (présents et à venir) ? L'idée d'une culture commune, par exemple, suppose de comprendre comment les désirs interagissent entre eux par le biais des mécanismes imitatifs. La portée à la fois éthique et politique de l'éducation se joue ici: que signifie "faire communauté", avoir une appartenance à quelque chose de commun? Or, la question du contenu, des choix de ce que l'on veut faire ensemble, est ontologiquement secondaire: s'il n'y a pas désir, il n'y aura que forcing et endoctrinement; peu importe alors que ce soit les "bons" choix s'ils sont imposés donc ne sont pas perçus et pensés comme "bons", comme "désirables" par les individus de la communauté.

Pour explorer ce problème, je prendrai comme partenaires Spinoza, Gabriel Tarde et René Girard: trois grands penseurs du rapport entre désir et imitation ${ }^{1}$.

1. Partenaires largement complémentaires à mon sens, même s'ils s'ignorent chronologiquement: Tarde ne fait jamais référence à Spinoza quand il construit son concept de désir, Girard a précisé 
L'articulation de ce rapport entre rapports (puisque désir et imitation sont des rapports) est cruciale dans la question de l'éducation: comment créer du "comme-un" parmi les individus, sans restreindre cet enjeu à un rapport unilatéral des plus anciens vers les plus jeunes? Pour répondre à cette question, il sera intéressant de distinguer deux formes du "proximal" donc de répondre à la question: qu'est-ce qu'être "proche” de quelqu'un?

\section{Le problème}

Pour penser l'articulation entre désir et imitation dans l'éducation, partons de Tarde. Ce dernier est fort optimiste sur les mécanismes de transmission du désir:

La curiosité ici mérite une place à part, sinon la place d'honneur. Tous les attroupements d'hommes qui finissent par opérer des révolutions, religieuses, politiques, artistiques, industrielles, commencent par se former sous l'empire de ce sentiment. Quand on voit une personne curieuse de n'importe quoi dont on se souciait naguère comme d'un fétu, aussitôt on devient désireux de connaître cette chose, et ce mouvement se propage très vite, et, à mesure qu'il se propage, l'intensité de ce désir croît en chacun par l'effet du mutuel reflet ${ }^{2}$.

Malheureusement, la théorie se heurte ici frontalement aux faits: si l'on prend l'exemple de la situation scolaire, tout professeur sait qu'il ne suffit pas de désir partager un savoir pour que les élèves l'imitent et se mettent eux-mêmes à désirer savoir.

Dans la perspective de l'apprentissage, la question du rapport entre désir et imitation est en effet capitale: suffit-il que je désire penser avec les élèves pour que je vaille modèle auprès d'eux, dont le désir se calquerait sur le mien et s'orienterait ainsi semblablement vers cet apprentissage? Du constat que «ça n'est pas aussi simple», que faut-il conclure? Dans cette perspective, deux interprétations du conflit entre la théorie et la pratique sont possibles.

a) Soit les faits sont véridiques: moi, professeur, j'aime ma matière et je ne comprends pas que cet amour ne soit pas lui-même désiré par les élèves. Donc, la théorie du désir mimétique de Girard est fausse.

b) Soit on donne sa chance à l'hypothèse girardienne: ce sont donc les faits qu'il faudrait réinterpréter. Si le mécanisme mimétique ne fonctionne pas, alors je dois prendre acte du peu de désir des élèves comme un symptôme de mon absence de désir pour ce que j'enseigne... C'est exactement le raisonnement que propose George Picard pour comprendre le désintérêt profond pour les joies de la lecture littéraire chez de nombreux individus ${ }^{3}$ :

que sa théorie du désir mimétique ne doit rien à Tarde - dont il estime peu les travaux. Cf.

R. Girard, Les origines de la culture [2004], Paris, Hachette littératures (Pluriel philosophie), 2006,

p. $189-190$ et 252 .

2. G. Tarde, Les lois de l'imitation, Paris, Les Empêcheurs de penser en rond - Seuil, 2001, p. 254.

3. Il rappelle que $42 \%$ des Français ne lisent jamais, selon les statistiques de l'INSEE. 
Cette réaction trouve évidemment ses causes dans l'enseignement calamiteux de la littérature qui semble avoir abandonné l'objectif de faire aimer la lecture (s'il l'a jamais eu). Créer du désir n'est certainement pas une tâche facile; je ne vois pas pourtant comment on peut ouvrir autrement l'accès aux œuvres. Dans ce domaine, la réussite repose sur une pédagogie mimétique. Si les humains désirent essentiellement par imitation, un objet devenant désirable par le truchement du désir d'un tiers (René Girard), il faut alors se rendre à l'évidence: les instituteurs et les professeurs sont rarement à la hauteur de cet enjeu affectif ${ }^{4}$.

Pour éclaircir le problème, distinguons l' "imitation", au sens technique défini par Tarde, et le "mimétisme", qui désignerait la reproduction à l'identique d'une attitude, selon le modèle de René Girard.

a) Pour Tarde, l'imitation est un processus fondamental de la vie sociale; savoir si elle est consciente ou non, volontaire ou non, spontanée ou réfléchie est secondaire:

je n'attache pas une capitale importance à cette division. Est-il vrai qu'à mesure qu'un peuple se civilise, sa manière d'imiter devienne de plus en plus volontaire, consciente, réfléchie? Je croirais plutôt l'inverse ${ }^{5}$.

Beaucoup plus important est de remarquer que l'imitation est moins ce qui se passe entre les individus que ce qui fait les individus. L'imitation fait circuler le désir "par" les individus. Le concept d'imitation défini par Tarde permet de penser la "transaction".

b) Alors que l'imitation s'exprime à travers les flux qui passent entre les individus, le mimétisme exprime les individus selon des gestes-miroirs. Le mimétisme articule les désirs "des" individus, il est un interactionnisme. Mon désir provient du mimétisme du désir de l'autre: ce mécanisme repose sur une triangulation des substances désirantes (les individus) et suppose la représentation (consciente ou non) du désir.

Suivons les analyses de Tarde, qui offrent de précieux outils, car le désir est un concept neutre car ontologique chez lui: il n'y a pas de sens à parler d'absence de désir au sens où il l'entend ${ }^{6}$. Le problème n'est pas «y a-t-il du désir? » mais « dans

4. G. Picard, Tout le monde devrait écrire, Paris, José Corti, 2006, p. 113-114. Les chiffres de l'INSEE sont cités par Picard à la note 21 de la page 87 du livre. Picard ne cite pas l'enquête, dont les termes exacts sont: « $42 \%$ d'entre elles [personnes interrogées de plus de quinze ans] sont en retrait vis-à-vis de cette pratique, soit qu'elles ne lisent jamais, soit que, n'attachant pas d'importance particulière à la lecture, la mémoire leur fasse défaut lorsqu'il s'agit de comptabiliser le nombre d'ouvrages lus au cours de l'année écoulée». Cf. H. Michaudon, «La lecture, une affaire de famille», Insee Première, $\mathrm{n}^{\circ} 777$, mai 2001.

5. G. Tarde, Les lois de l'imitation, p. 250-251.

6. Cf. G. Tarde, Les lois sociales, chapitre 1: «L'énergie de tendance psychique, d'avidité mentale, que j'appelle le désir, est, comme l'énergie de saisissement intellectuel, d'adhésion et de constriction mentale, que j'appelle la croyance, un courant homogène et continu qui, sous la variable coloration des teintes de l'affectivité propre à chaque esprit, circule identique, tantôt divise, éparpillé, tantôt concentre, et qui, d'une personne à une autre, aussi bien que d'une perception à une autre 
quelle direction coule le désir?». L'intérêt de cette perspective est de nous forcer à continuer de chercher, échappant ainsi à la condamnation de Picard mais aussi à l'abandon des thèses girardiennes; le problème devient de savoir ce que désire le professeur qui dit «aimer sa matière»... Peut-être désire-t-il quelque chose en elle qui est rétif à la circulation, au partage? De plus, le concept de désir est nécessairement réciproque chez Tarde. Il ne suit pas le schéma fortement individué de Girard, au sein duquel les rapports de désir sont souvent unilatéraux (désir du modèle $)^{7}$. Dès lors, le problème n'est pas seulement que les élèves désirent faire comme le professeur; la réciproque est également intéressante: le professeur imite les désirs de ses élèves.

C'est en effet une expérience quotidienne: le désir du professeur est également alimenté par celui des élèves. Ainsi, lorsque les élèves ne désirent pas être là où ils sont (présentement: en classe), le désir du professeur d'être là, avec eux, tend à diminuer. Cet ajustement mutuel des désirs est une expérience banale, mais on n'en tire peut-être pas toutes les conséquences qu'on devrait. En effet, les analyses pédagogiques interrogent souvent le problème de l'intérêt des élèves, de leur désir, de leur motivation; mais pourquoi n'y a-t-il aucune étude sur l'intérêt des professeurs, leur désir? Pourquoi pas la question: «comment intéresser les enseignants à ce que les élèves peuvent leur apporter?», au moins aussi importante que celle portant sur l'intérêt des élèves à apprendre. Va-t-il de soi qu'un professeur arrive dans sa classe chargé de désir et d'amour? L'inverse se vérifie plus souvent. Dans ces conditions, une interrogation sur le seul intérêt des élèves est un problème mal posé, donc voué à des alternatives biaisées et à des solutions bancales... Tout l'enjeu de la création du "comme-un" dans l'éducation trouve ici un point aveugle: c'est toujours le collectif gérontocratique qui veut le bien des jeunes individus en se posant comme modèle à mimer.

D’après les caractéristiques tardiennes du désir, une nouvelle hypothèse émerge: le désir d'un enseignant peut être stérile dans la mesure où l'imitation de ce désir ne suscite aucune disposition active chez les élèves. Si l'imitation du désir est une théorie vraie, alors je peux susciter le désir d'apprendre à penser uniquement si je désire moi-même apprendre "de" l'autre. Dans tous les cas, il y a du désir; celui-ci circule plus ou moins, dans des directions diverses, plus ou moins concentré ou éparpillé, plus ou moins réciproquement. Ce qu'il faut comprendre, c'est que passe nécessairement entre le professeur et les élèves du désir. Le problème n'est pas celui de l'existence du désir, mais de son parcours; c'est la question du sens: la "signification" du désir se lit aux "orientations" qu'il prend. Par exemple, si le professeur désire que les élèves écoutent la vérité qu’il leur annonce, les élèves

dans chacune d'elles, se communique sans altération», disponible sur: http://classiques.uqac.ca/ classiques/tarde_gabriel/les_lois_sociales/les_lois_sociales.html.

7. La notion de médiation «double» ou «réciproque» ne change rien à l'affaire: elle n'est qu'un emballement du processus de la rivalité mimétique, une fois qu'on ne sait plus qui a commencé à imiter. Cela devient une succession de mimétismes, toujours unilatéraux en eux-mêmes. Cf. R. Girard, Mensonge romantique et vérité romanesque, Paris, Grasset, 1961, p. 163, 195 et 199-200. 
seront traversés par le désir d'être passif. Il y a donc bien imitation du désir: le comportement des élèves ne fait que révéler le sens du désir de l'enseignant. Dans ce cas, les élèves ne recevront qu'une faible quantité de désir d'être actifs; ce sont donc les autres désirs qui les feront agir - désir de bavarder, désir d'être ailleurs. En revanche, si l'enseignant désire construire avec eux quelque chose, les élèves seront traversés par un désir de faire.

Par conséquent, un professeur disant aimer sa matière, mais se heurtant à l'impassibilité des élèves, est peut-être pris dans cette double impasse qui pourrait expliquer son aporie: il désire "transmettre" des solutions "aux" élèves. Les deux écueils sont corrélatifs: son désir est orienté sur les solutions, et non sur les problèmes, donc il croit ne pas avoir besoin d'être pris lui-même dans un rapport désirant imitatif avec les élèves. Ce pseudo-désir comme volonté d'avoir des réponses - pour remplir le manque du non-savoir - est souvent né dans la honte (de ne pas savoir) et conduit à l'orgueil (de savoir). Comment, dans ces conditions, créer le désir des problèmes chez les autres s'il est vrai que n'aiment engendrer que ceux qui ont aimé leur propre engendrement ${ }^{8}$ ? Ce désir biaisé ne peut générer chez les élèves qu'un mime superficiel des réponses, entraînant de tristes mascarades. Dans les cours, on voit alors les élèves se livrer à un mime de la forme du savoir, véritable posture d'allégeance à des pratiques qu'ils tentent maladroitement de reproduire afin de satisfaire une attente floue présumée, pour coller au désir du professeur.

À l'inverse, désirer "apprendre" des problèmes “avec" les élèves conduit à vivre un désir orienté vers une construction mutuelle. Les élèves peuvent imiter le désir du professeur car ce désir appelle une telle circulation, c'est-à-dire que le professeur lui-même est prêt à imiter leurs désirs - plutôt que les rectifier a priori ${ }^{9}$. Je ne dis pas: «se calquer sur leurs désirs», je dis bien : «imiter également», ce qui entraîne une correction mutuelle des désirs, un apprentissage réciproque. Employons une image d'ingénierie des fluides: soit vous versez de l'eau dans quelque chose, vous finissez par créer un réservoir et l'objet se met à flotter dans le meilleur des cas, sinon il coule; soit vous injectez de l'eau vers quelque chose, et acceptez d'en récupérer en retour, et vous créez un circuit.

\section{La condition de circulation du désir : ressemblance ou similitude}

a) Chez René Girard, une des conditions de la rivalité mimétique est la ressemblance (première forme du proximal), cela concerne la représentation du désir : si le modèle est suffisamment proche (médiation interne), il y a rivalité, fascination haineuse, envie, etc.; si le modèle est éloigné (dans l'espace, le temps, ou symboliquement), il n'y a pas rivalité (médiation externe, ex: le Christ). Dans cette perspective, le

8. M. Serres, Le Tiers-Instruit, Paris, Gallimard (Folio), 2004, p. 93.

9. Le désir voué à l'impasse, c'est le désir “orthopédique". Cette volonté de redresser les esprits et les corps sans désir d'aucune réciprocité provient de la croyance en un pouvoir fondé sur une vérité détenue "une fois pour toutes". 
peu de ressemblance est salutaire. Paradoxalement, il semblerait que le modèle de Girard puisse offrir une critique du modèle de l'humanisme abstrait qui met en concurrence affective les individus alors qu'il prétend faire leur bien (l'égalité de droit se transformant en méritocratie). La passivité ainsi générée ne provient pas de ce qu'on imite - puisqu'on ne peut pas faire autre chose - mais du fait de l'imitation d'un modèle individué (c'est-à-dire préalablement institué et défini) et semblable à soi (tel qu'on s'imagine vouloir être, imagination conditionnée par ce que d'autres ont défini et réussi à nous faire désirer). On est dans le cadre d'individus en relation dans un collectif ayant instauré des modèles.

b) Spinoza met au cœur du rapport entre désir et imitation la notion de similitude (deuxième forme du proximal). L'émulation (si l'autre désire $x$, alors je désire $x$ ) a lieu à condition que les individus partagent suffisamment de propriétés communes pour former une nouvelle heccéité. Ici, la perspective est toute différente : l'imitation du désir permet de (se) construire avec un partenaire d'individuation similaire à soi. Alors que la ressemblance concerne la représentation du désir, la similitude est une condition de circulation du désir. Le problème n'est pas interindividuel: la question n'est pas que certains imitent le désir d'autres; la condition de l'éducation est transindividuelle: pour que des rapports soient éducatifs (au sens large et émancipateur), il est nécessaire qu'ils fassent circuler du désir. C'est une question de flux et non de poses. En fait, on n'imite rien à proprement parler: le désir de l'autre agit sur nous comme une puissance effective qui produit un écart, un clinamen sur notre propre système de pensées. On est dans le cadre de processus d'individuation (il y a de l'individuel) qui forment des moments de "comme-un", et se forment par des moments de "comme-un".

La problématique de Girard permet d'analyser le devenir passif: en cela elle est précieuse anthropologiquement, mais elle ne peut être d'aucun secours pour penser les finalités politiques de l'éducation car elle conduit à des faux sauvetages pédagogiques comme de bénir le concept de motivation. À l'inverse, Spinoza inscrit explicitement la formation du désir dans le cadre d'un conatus collectif: le désir peut circuler si les individus constituent effectivement une heccéité propre, c'est-à-dire se forment mutuellement par individuations réciproques. Cette identification réciproque les uns aux autres (la similitude comme conquête: comment puis-je composer mes rapports avec l'autre?) décrit un mouvement, un processus dynamique; elle est donc étrangère à la question de l'identité (la ressemblance comme constat: me ressemble-t-il ?), toujours trop statique au regard des processus d'individuation qui nous traversent en permanence. Cette nécessité de "réciprocité anthropologique" suppose un réquisit d'égalité épistémique: une telle immanence éthique est sans doute la condition pour réaliser ontologiquement quelque chose comme un réel apprentissage réciproque - qui ne soit pas qu'un vœu pieux.

Ce point est capital et il faut en comprendre la conséquence simple: lorsque je dis "désirer partager" un savoir, il est possible que je désire en fait "donner" ce savoir à d'autres en même temps que je ne désire rien "recevoir" de ces mêmes autres. Par exemple, je partage mon amour pour Molière, mais je ne suis pas intéressé par les goûts de ceux à qui j'évoque ce désir de lire la grande comédie 
critique. En effet, il n'est pas difficile d'imaginer un parent ou un professeur qui pense avoir à apprendre “à” l'enfant en même temps qu'il estime n'avoir rien à apprendre "de" cet enfant. Dès lors, si l'on prend à la lettre le mécanisme d'imitation du désir, la conséquence est la suivante: si l'enfant imite le désir de l'adulte, alors il désire lui donner pareillement quelque savoir ou savoir-faire. Mais le désir de l'adulte rend cette réciproque impossible. On a donc ici une structure de désir proprement contradictoire: le désir de l'adulte crée des dispositions imitatives de désir (vouloir donner) chez l'enfant mais refuse qu'elles adviennent - car il ne désire pas recevoir : par mépris ou indifférence de la culture adolescente par exemple. Il bloque donc la circulation du désir : répétée plusieurs fois, on imagine que cette situation peut créer des dispositions paradoxales chez l'enfant et une frustration qui le conduiront à ne pas vouloir recevoir (lui non plus) ce que l'autre espère ou prétend lui apporter.

Insistons: le véritable enjeu dans les rapports entre imitation et désir est celui de la circulation du désir. La question de l'objet du désir devient secondaire: importent d'abord les conditions de circulation en tant qu'elles rendent possible la curiosité intellectuelle et l'éducation continuée - donc la formation de soi tout au long de la vie. La critique célèbre de l’objectivité du désirable par Spinoza ne fait donc pas s'écrouler la perspective de s'intéresser aux conditions de création du désirable dans l'éducation ${ }^{10}$. L'objectif n'est pas de créer un dispositif désirable, mais de se rendre commun aux élèves en les invitant à participer avec soi à une aventure d'idée où nous soyons "comme un". En effet, une individuation de la connaissance faite ensemble ne peut que signifier une individuation ontologique commune. Autrement dit, apprendre à penser n'est possible que par la mise en place de la circulation du désir, c'est-à-dire l'apprentissage de la "convenance" les uns aux autres. De fait, chez Spinoza la notion de convenance n'est rien moins que ce qui fonde une communauté entre individus agissants, actifs au sens plein du terme; la convenance permet donc le déploiement de l'activité rationnelle. Et qui, sinon le professeur, peut faire le premier geste vers cette convenance mutuelle? Cela implique un per communis minimum: les différences avec lesquelles chacun arrive dans la classe permettent une tension féconde (métastabilité) si et seulement si chacun se prête au jeu de l'implication mutuelle dans la situation partagée. La condition éthique de l'éducation consisterait à utiliser sa différence pour se rendre commun aux élèves - et non la légitimer (rationalisation), s'en enorgueillir (névrose), brefl'instancier. La circulation du désir suppose que tous nous convenions les uns

10. Appliqueé à l'éducation, la proposition 9 du livre III de l'Éthique dit ceci: ce n'est pas parce qu'une situation d'apprentissage est désirable que je désire apprendre, c'est parce que je désire apprendre que la situation d'apprentissage me paraît désirable. L'origine purement subjective du désir semblait donc rendre vaine toute tentative de vouloir créer des conditions objectives du désirable. À tout le moins, l'ordre causal décrit par Spinoza peut rendre attentif aux dérives pédagogiques qui, croyant proposer des situations «objectivement désirables», ne font que répéter le désir conditionné socialement et historiquement; par exemple, un cours aux séquences très courtes peut apparaître plus désirable aux élèves uniquement parce que cela reproduit la rythmicité des médias contemporains. 
aux autres, c'est-à-dire que la relation pédagogique refonde et modifie les acteurs de cette relation. Tout se passe à travers les individus plus qu'à partir d'eux. La différence entre le professeur et les élèves n'est productrice que si chacun se risque à la construction collective, que si est fait le pari de l'individuation "avec", c'est-àdire "à travers", les élèves.

Pascal Sévérac démontre bien cette primauté du vocabulaire relationnel chez Spinoza: ce risque est la «seule condition pour qu'un mode fini soit actif» car «il faut et il suffit que ce à travers quoi ce mode détermine un effet soit identique à ce $a ̀$ travers quoi il est déterminé à produire cet effet». Il en découle qu' "être contraint, pour un mode fini, signifie donc toujours en quelque manière être déjà actif - agir en vertu de sa participation à l'activité même" de cet autre qui agit à travers lui ${ }^{11}$. La circulation du désir devient le signe du fonctionnement bénéfique - i.e. commun - pour les acteurs de l'apprentissage: elle signale la présence de l'énergie nécessaire à l'individuation, donc que l'individuation a lieu, donc qu'un problème est effectivement construit par les individus en train de se modifier par cette épreuve.

Il est intéressant de remarquer que le fait de chercher les conditions concrètes de circulation du désir conduit aux perspectives antiques sur la sagesse. En effet, si on pose précisément la question: «comment créer de la similitude pour un parent ou un enseignant?», il faut reconnaître que les analyses proposées n'offrent pas de solution immédiate. Elles renvoient bien plutôt à la notion de formation d'habitudes, à la nécessité de toute une préparation en amont. La similitude ferait partie de ces «effets essentiellement secondaires » : la vouloir au moment où l'on en a besoin est se condamner à échouer ${ }^{12}$. Penser les rapports entre désir et imitation nous mène paradoxalement vers une éthique de la patience. Si désirer mimer le semblable est souvent le stigmate d'une avidité qui «veut tout de suite», désirer imiter par la conquête de la similitude suppose au contraire toute une expérience de la formation de soi - avec le temps long que cela suppose. Ici, le problème de la conquête éthique de la similitude est synchroniquement opposé à la question kantienne de la moralité: je ne peux pas décider de réagir à l'événement au moment où il advient - en suivant l'impératif catégorique comme test de la non-immoralité

11. Cf. P. Sévérac, Le devenir actif chez Spinoza, Paris, Honoré Champion, 2005, p. 89-91 (souligné par l'auteur).

12. Une anecdote célèbre du rapport de Kant à son valet Lampe offre un bel exemple d'effet essentiellement secondaire: «Et je trouvai un trait touchant du regret qu'éprouva Kant pour son vieux serviteur qui n'avait jamais rien valu, qui est inscrit dans son carnet. D'autres personnes notent ce dont elles désirent se souvenir. Là, Kant avait noté ce qu'il devait oublier : Mem. - février 1802 - il ne faut plus se souvenir du nom de Lampe». Oublier quelqu'un: le geste de Kant est paradoxal car vouloir oublier quelqu'un est le meilleur moyen pour s'ancrer dans la mémoire son souvenir, donc faire échouer le projet d'oubli. Vouloir dormir est tout aussi inefficace: s'efforcer de dormir au moment où l'on veut s'endormir est le meilleur moyen pour s'énerver et voir s'éloigner la possibilité de s'endormir effectivement. Dans les deux cas, il faut donc se préparer à l'événement, c'est-à-dire agir chaque jour en vue d'acquérir des dispositions qui me permettent de composer mes rapports avec les rencontres de ma vie à l'occasion de leur surgissement. Cf. T. de Quincey, Les derniers jours d'Emmanuel Kant, Paris, Allia, 2004. Le concept d' " effet essentiellement secondaire» fut proposé par J. Elster dans Le laboureur et ses enfants, Paris, Minuit, 1987. 
de mon action. On est beaucoup plus proche de l'éthique de l'habitude d'Aristote: quand l'événement advient, il est toujours "trop tard" si je ne me suis pas préparé à sa possibilité. Par contraste, conquérir la similitude avec les autres fonctionne comme transformation de soi effective donc me rend capable de réagir à l'événement sans y penser au moment où il advient. La conquête de la similitude conduit ultimement à pouvoir faire circuler son désir (en acceptant d'être pris dans celui de l'autre) par une transformation de soi qui rend «non volontaire» l'efficace du geste pédagogique au moment où il doit avoir lieu. On remarquera que tout ceci est déjà dans Platon, lorsqu'Alcibiade, ivre, rend hommage à Socrate à la fin $\mathrm{du}$ Banquet: Socrate, sa vie, ses choix, sont suprêmement désirables parce qu'il ne cherche jamais, dans ses interactions avec les jeunes gens, à être désirable ou à se rendre semblable à eux. Son pouvoir découle de sa manière de vivre, construite au jour le jour. Socrate n'est jamais semblable aux jeunes gens, mais il sait devenir suffisamment similaire à eux pour que quelque chose circule: le désir.

Par opposition à cette structure temporelle patiente, qui suppose de la part de l'éducateur d'accepter d'être transformé par la rencontre (c'est-à-dire le désir de l'autre), je voudrais souligner un effet nuisible et courant des situations didactiques. La trace la plus patente et la plus caricaturale de la volonté empressée d'aller vers les élèves se remarque dans ce que j'appellerais l' "effet Dupondt", en hommage au racisme burlesque des jumeaux inventés par Hergé. Ceux-ci ont une propension à se déguiser à la manière de ce qu'ils croient être la culture des autres; ils veulent ainsi paraître comme les Chinois, en devenir les semblables, mais leurs propres clichés ne font que trahir plus bouffonnement le gouffre qui les en sépare. Par exemple, en didactique de la philosophie, on remarque ainsi des initiatives pour plaire aux élèves en leur offrant ce qu'on suppose être de leur goût. Avec une certaine maladresse et un peu de condescendance, l'usage d'un objet "artistique" en cours devient prétexte à faire "ensuite" de la vraie philosophie, et vaut comme un gage de bonne volonté - "vous n'allez peut-être pas aimer ce qu'on va faire ensuite, mais vous devez reconnaître que j'ai été sympathique de faire ça avant». Sur le site pédagogique de l'académie d'Amiens, on trouve un exemple des détours didactiques qui veulent illustrer, rendre sympathique la philosophie ${ }^{13}$. Afin d'ajouter un peu de couleur et de chaleur aux idées «forcément difficiles» de la philosophie, un professeur raconte qu'il se sert de Tintin ou d'Astérix. Il distribue une planche - sectionnant arbitrairement des œuvres narratives dont la trame est l'une des principales forces - pour ensuite donner des textes à lire ${ }^{14}$. Ce prélude

13. Disponible sur Internet: http://pedagogie.ac-amiens.fr/philosophie/sujets/bd.html.

14. Rabattre le génie narratif d'Hergé à une scène illustrant le concept de raison chez Kant (de manière aberrante), ou bien canaliser le génie humoristique de Goscinny vers une pédante analyse sur «l'esclave Maître de la Nature après avoir été Esclave de la Nature, donc bientôt Maître "médiat" du Maître", n'est pas rendre justice à ce qui peut fonctionner comme art dans leurs œuvres. De manière générale, la bande dessinée offre un cas exemplaire des mésusages de l'art par la pédagogie. Quasiment tous les livres de pédagogie voulant «tirer» quelque chose de l'amour des jeunes pour la bande dessinée - énorme cliché! - sont médiocres et témoignent d'une grande méconnaissance du médium. À aucun moment les pédagogues ne croient à la puissance critique du 
sympathique est la politesse du non-pédagogique. Pour reprendre les termes de Michel Fabre, un tel dispositif n'est qu'une «juxtaposition d'un problème incitatif visant à motiver les élèves et d'une leçon magistrale ou dialoguée», mais il n'ouvre aucune véritable perspective sur le fonctionnement lui-même de la pensée ${ }^{15}$.

Penser en termes d'individuel et de "comme-un", dans l'entrelacs des désirs des uns et des autres s'imitant les uns les autres, permet de prendre en compte les individuations en train de se faire, plutôt que de partir d'individus déjà individués - ni du côté de ceux qui enseignent, ni du côté de ceux qui apprennent. Les bonnes habitudes politiques (créer ensemble de l'avenir) s'acquièrent dès l'éducation, et tout au long de la vie (éducation continuée). Continuer à apprendre "pour devenir" suppose de faire de chaque situation éducative l'occasion de créer de la similitude, c'est-à-dire de se transformer. Je ne vois pas d'autre sens cohérent pour définir la Bildung. La formation de l'individuel que je suis a lieu à chaque occasion de faire "comme-un" avec les autres. Il me semble que faire l'expérience de ce devenir “comme-un" est ce qui donne un sens véritablement politique à l'éducation en vue de vivre en société.

Sébastien CHARbONNIER

CREN

Université de Nantes

support; ils opèrent un instrumentalisme pur en se servant transitoirement d'un support supposé aimé des jeunes pour mieux les emmener ailleurs. Sur la pauvreté de la littérature pédagogique traitant de la bande dessinée, cf. le compte rendu critique exhaustif de la littérature secondaire sur la littérature dessinée, sévère mais juste, de H. Morgan et M. Hirtz, Le petit critique illustré, Montrouge, PLG, 2005.

15. Cf. la conclusion de l'article de $\mathrm{M}$. Fabre et $\mathrm{A}$. Musquer, «Comment aider les élèves à problématiser? Les inducteurs de problématisation ", Les Sciences de l'Éducation - Pour l'Ère Nouvelle, 3, 2009, p. 111-127 (je souligne). 